\title{
Fast and Easy Computation of Approximate Smallest Enclosing Balls
}

\author{
Thomas Martinetz, Amir Madany Mamlouk \\ Institute for Neuro- and Bioinformatics \\ University of Lübeck \\ D-23538 Lübeck, Germany \\ martinetz@informatik.uni-luebeck.de
}

\author{
Cicero Mota \\ Departamento de Matematica \\ Universidade Federal do Amazonas \\ 69.077-000,Manaus, Brazil \\ mota@ufam.edu.br
}

\begin{abstract}
The incremental Badoiu-Clarkson algorithm finds the smallest ball enclosing $n$ points in d dimensions with at least $\mathcal{O}(1 / \sqrt{t})$ precision, after $t$ iteration steps. The extremely simple incremental step of the algorithm makes it very attractive both for theoreticians and practitioners. A simplified proof for this convergence is given. This proof allows to show that the precision increases, in fact, even as $\mathcal{O}(u / t)$ with the number of iteration steps. Computer experiments, but not yet a proof, suggest that the u, which depends only on the data instance, is actually bounded by $\min \{\sqrt{2 d}, \sqrt{2 n}\}$. If it holds, then the algorithm finds the smallest enclosing ball with $\epsilon$ precision in at most $\mathcal{O}\left(n d \sqrt{d_{\mathrm{m}}} / \epsilon\right)$ time, with $d_{\mathrm{m}}=\min \{d, n\}$.
\end{abstract}

Key words: computational geometry, smallest enclosing ball, pattern recognition

\section{Introduction}

The smallest enclosing ball (SEB) is a classical problem in computational geometry. In its simplest formulation a set $S$ of $n$ points $\mathbf{x}$ in $\mathbb{R}^{d}$ is given, the goal is to find the ball with smallest radio containing $S$. It appears frequently as an intermediate step in many applications, e.g., the tuning of Support Vector Machine parameters [5], gap-tolerant classifiers [4], k-center clustering [2], hand-recognition [3], facerecognition [11] or the detection of genetic binding sites [8], among others.

There are elaborated combinatorial algorithms which provide exact solutions, also in high dimensions (see e.g. $[13,15,6])$, but without a polynomial worst-case bound. Approximate solutions can be obtained in polynomial time, e.g., via core-sets, see e.g., [1, 10]. Much simpler for obtaining approximate solutions in polynomial time and, hence, interesting for the practitioner, is the algorithm introduced by Badoiu and Clarkson [1].

\section{The Badoiu-Clarkson algorithm}

The algorithm is an iterative three-lines procedure and works as follows:

1. let $\mathbf{c}_{t}$ be the guess for the center at step $t$, set $\mathbf{c}_{0}=\mathbf{0}$;

2. at step $t$, choose $\mathbf{x}_{t}$ as the point of $S$ which is furthest away from $\mathbf{c}_{t}$;

3. iterate according to

$$
\mathbf{c}_{t+1}=\mathbf{c}_{t}+\frac{1}{1+t}\left(\mathbf{x}_{t}-\mathbf{c}_{t}\right) .
$$

At every step, the algorithm moves the current center, towards the furthest point, by a fraction of the radius of the actual approximation of the SEB. Figure 1 illustrates the algorithm. To better understand how the algorithm works, we note that the iteration step can be rewritten as

$$
\mathbf{c}_{t+1}=\frac{1}{t+1}\left(\mathbf{x}_{0}+\mathbf{x}_{1}+\cdots+\mathbf{x}_{t}\right) \text {. }
$$

Thus, the Badoiu-Clarkson algorithm is actually a method that selects points of $S$ in such a way that the barycentre of the selected points converges against the center of the minimal enclosing ball.

Formula (2) is easily obtained by setting

$$
\mathbf{u}_{t}=t\left(\mathbf{c}_{t}-\mathbf{c}\right) .
$$

where $\mathbf{c}$ denotes the unknown center of the SEB of $S$. By plugging the iteration rule in (1) into Formula (3), we find

$$
\mathbf{u}_{t+1}=\mathbf{u}_{t}+\mathbf{x}_{t}-\mathbf{c},
$$

which implies Formula 2.

Theorem 1 states the convergence of the algorithm. It was proven in [1]. Here we give a simplified proof based on the following lemma [7]. Since the lemma has a short proof, it is include here for completeness.

Lemma 1 Let $S$ be a set of points in $\mathbb{R}^{d}$ and $B$ its smallest enclosing ball, then any closed half-space that includes the center of $B$ also includes a point on the surface of $B$. 
Proof Let $\mathbf{c}$ and $R$ be center and radius of $B$, and $H$ a closed half-space including $\mathbf{c}$. We can suppose without loss of generality that $\mathbf{c}=0$ and the normal to the border of $H$ pointing outside is $\mathbf{e}_{1}$. By contradiction, assume the statement is false. In this case, for every x, either $S \cap H$ and $|\mathbf{x}|<R$, or $\mathbf{e}_{1} \cdot \mathbf{x}>0$ is valid. This implies the existence of a $\epsilon>0$ such that $\left|\mathbf{x}-\epsilon \mathbf{e}_{1}\right|<R$, for every $\mathbf{x}$ in $S$, contradicting the minimality of $B$.

Theorem 1 Let $S$ be a finite subset of $\mathbb{R}^{d}$, $\mathbf{c}$ and $R$ be the center and radius of the smallest enclosing ball for $S$, then for every step $t$

$$
\frac{\left|\mathbf{c}_{t}-\mathbf{c}\right|}{R} \leq \frac{1}{\sqrt{t}} \quad \text { and } \quad \frac{\left|R_{t}-R\right|}{R} \leq \frac{1}{\sqrt{t}},
$$

with $R_{t}$ as the radius of the smallest enclosing ball with cen$\operatorname{ter} \mathbf{c}_{t}$.

Proof Without loss of generality we can set $\mathbf{c}=\mathbf{0}$ and $R=1$. We introduce

$$
\mathbf{u}_{t}=t \mathbf{c}_{t}
$$

Then, the iteration rule (1) can be written as

$$
\mathbf{u}_{t+1}=\mathbf{u}_{t}+\mathbf{x}_{t}
$$

and the change of the length of $\mathbf{u}_{t}$ obeys

$$
\mathbf{u}_{t+1}^{2}-\mathbf{u}_{t}^{2}=2 \mathbf{u}_{t} \cdot \mathbf{x}_{t}+\mathbf{x}_{t}^{2}
$$

By Lemma 1 , there is always at least one point $\mathrm{x} \in S$ on the closed half-sphere of the SEB opposite to the actual estimate $\mathbf{c}_{t}$. Hence, for $\mathbf{x}_{t}$ as the point of $S$ which is most distant from $\mathbf{c}_{t}$ always

$$
\mathbf{u}_{t} \cdot \mathbf{x}_{t} \leq 0
$$

is valid. Thus we obtain

$$
\mathbf{u}_{t+1}^{2}-\mathbf{u}_{t}^{2} \leq 1
$$

and, hence, the length of $\mathbf{u}_{t}$ is bounded by

$$
\left|\mathbf{u}_{t}\right|^{2} \leq t
$$

For the convergence of $\mathbf{c}_{t}$, this yields

$$
\frac{\left|\mathbf{c}_{t}-\mathbf{c}\right|}{R}=\frac{\left|\mathbf{u}_{t}\right|}{t} \leq \frac{\sqrt{t}}{t}=\frac{1}{\sqrt{t}} .
$$

\section{1. $\mathcal{O}(u / t)$ convergence}

Next, we are going to show that in fact the precision of the solution increases like $\mathcal{O}(u / t)$ with the number of iteration steps.

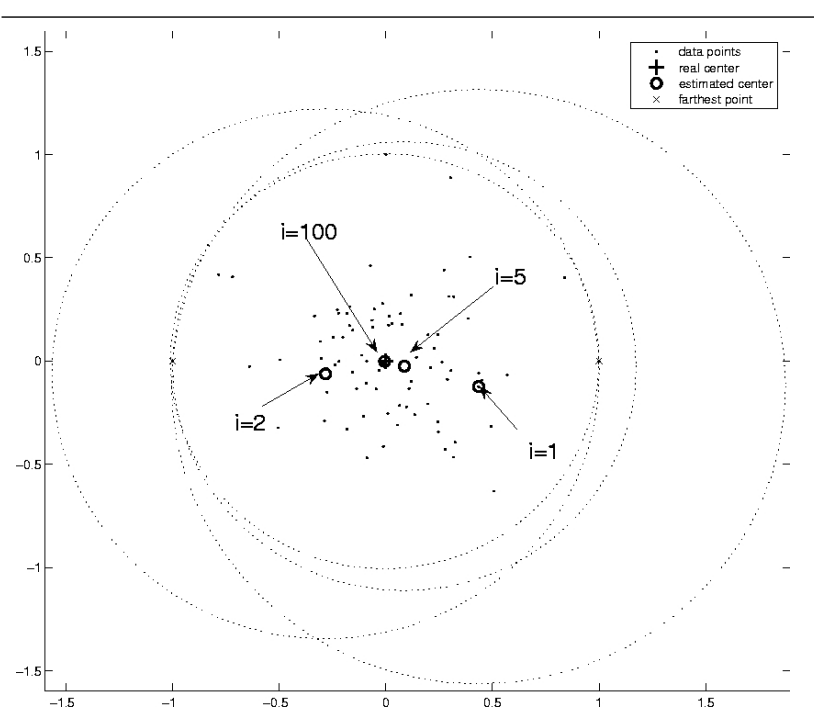

Figure 1. Example run for 80 normal distributed points on a plane. The approximated center $c_{t}$ and thus the enclosing ball converges against the real center $\mathrm{c}$, shown here after $1,2,5$, and 100 iterations.

Theorem 2 Let $S$ be a finite subset of $\mathbb{R}^{d}$, c and $R$ be the center and radius of the smallest enclosing ball for $S$, then there is a positive number $u=u(S)$ such that for every step $t$

$$
\frac{\left|\mathbf{c}_{t}-\mathbf{c}\right|}{R} \leq \frac{u}{t} \quad \text { and } \quad \frac{\left|R_{t}-R\right|}{R} \leq \frac{u}{t},
$$

with $R_{t}$ as the radius of the smallest enclosing ball with cen$\operatorname{ter} \mathbf{c}_{t}$.

Proof As in the proof of Theorem 1, we can set $\mathbf{c}=\mathbf{0}$ and $R=1$ without loss. The stated convergence rate is achieved if $\mathbf{u}_{t}$ stays bounded (see eq. (12)). This is indeed the case. Let $S^{\prime}$ be the set of points of $S$ on the surface of the SEB and $\alpha$ the distance between $S^{\prime}$ and $S-S^{\prime}$. Theorem 1, together with Lemma 1, allow to conclude that after

$$
t_{\alpha}=1+1 / \alpha^{2}
$$

iterations each $\mathbf{x}_{t}$ will lie on the surface of the SEB, i.e., $\left|\mathbf{x}_{t}\right|=1$. With $\mathbf{u}_{t}^{\prime}$ we introduce the projection of $\mathbf{u}_{t}$ onto the subspace $E^{\prime}$ spanned by these $\mathbf{x} \in S^{\prime}$. If $\mathbf{u}_{t}^{\prime}$ stays bounded, then also $\mathbf{u}_{t}$. From $t_{\alpha}$ on, the $\mathbf{x}_{t} \in S$ for which $\left(\mathbf{x}_{t}-\mathbf{c}_{t}\right)^{2}$ is maximal is the $\mathbf{x}_{t} \in S^{\prime}$ for which $\left(\mathbf{x}_{t}-\mathbf{c}_{t}\right)^{2}=$ $\mathbf{x}_{t}^{2}+\mathbf{c}_{t}^{2}-2 \mathbf{u}_{t} \cdot \mathbf{x}_{t} / t$ is maximal, or equivalently, for which $\mathbf{u}_{t} \cdot \mathbf{x}_{t}=\mathbf{u}_{t}^{\prime} \cdot \mathbf{x}_{t}$ is minimal. Lemma 1 states that $\mathbf{u}_{t}^{\prime} \cdot \mathbf{x}_{t} \leq 0$. Before proceeding, let's set

$$
-\delta=\max _{\left|\mathbf{u}^{\prime}\right|=1} \min _{\mathbf{x} \in S^{\prime}}\left\{\mathbf{u}^{\prime} \cdot \mathbf{x}\right\}
$$

We now discriminate two cases: 
1) $\delta>0$

2) $\delta=0$.

Note that $\mathbf{u}^{\prime}$ with $\left|\mathbf{u}^{\prime}\right|=1$ varies only within the subspace $E^{\prime}$. For case 1 ), it can easily be proven that $\mathbf{u}^{\prime}{ }_{t}$ remains bounded. Case 2) is a little bit more tedious.

1) For each iteration step, $\mathbf{u}_{t}^{\prime} \cdot \mathbf{x}_{t} \leq-\delta\left|\mathbf{u}^{\prime}{ }_{t}\right|$. Analogously to Equation (8), we obtain

$$
\begin{aligned}
\mathbf{u}_{t+1}^{\prime 2}-\mathbf{u}_{t}^{\prime 2} & =2 \mathbf{u}_{t}^{\prime} \cdot \mathbf{x}_{t}+\mathbf{x}_{t}^{2} \\
& \leq-2 \delta\left|\mathbf{u}_{t}^{\prime}\right|+1
\end{aligned}
$$

The negative contribution to the change of $\left|\mathbf{u}^{\prime}\right|_{t}$ increases with $\left|\mathbf{u}_{t}^{\prime}\right|$ and keeps it bounded. Even more, since after $t_{\alpha}$ iterations $\left|\mathbf{u}_{t}^{\prime}\right|$ decreases as soon as it becomes larger than $1 / 2 \delta$, it holds

$$
\left|\mathbf{u}_{t}^{\prime}\right| \leq \max \left\{\left|\mathbf{u}_{t_{\alpha}}^{\prime}\right|, \frac{1}{2 \delta}+1\right\} .
$$

2) If $\delta=0$, the set

$$
U=\left\{\mathbf{u} \in E^{\prime} ;|\mathbf{u}|=1 \text { and } \min _{\mathbf{x} \in S^{\prime}}\{\mathbf{u} \cdot \mathbf{x}\}=0\right\}
$$

is non-empty. Therefore, $S^{\prime}$ is contained in the convex set of $E^{\prime}$ determined by

$$
\mathbf{u} \cdot \mathbf{x} \geq 0, \quad \forall \mathbf{u} \in U
$$

At least one of the previous inequalities is strict, otherwise $S^{\prime}$ would be a proper subset of $E^{\prime}$. Therefore, the sets

$$
S^{\prime \prime}=\left\{\mathbf{x} \in S^{\prime} \mid \mathbf{u} \cdot \mathbf{x}=0 \text { for all } \mathbf{u} \in U\right\}
$$

and $S_{\mathrm{c}}^{\prime \prime}:=S^{\prime}-S^{\prime \prime}$ are linearly separable by a maximal positive margin $\Delta[9,12]$. That is, there is an unity vector $\mathbf{v}$ in $E^{\prime}$ such that

$$
\mathbf{v} \cdot \mathbf{x}=0 \quad \forall \mathbf{x} \in S^{\prime \prime} \quad \text { and } \quad \mathbf{v} \cdot \mathbf{x} \geq \Delta \forall \mathbf{x} \in S_{\mathrm{c}}^{\prime \prime} .
$$

$\mathbf{u}^{\prime}$ changes according to the MinOver learning rule [9, 12], a slight modification of the perceptron learning rule [14]. Therefore, after a finite number of learning steps, $\mathbf{x}_{t}$ will always be an element of $S^{\prime \prime}$. Then the $\mathrm{x}_{t} \in S^{\prime}$ that minimizes $\mathbf{u}_{t}^{\prime} \cdot \mathbf{x}$ is identical to the $\mathbf{x}_{t} \in S^{\prime \prime}$ that minimizes $\mathbf{u}^{\prime \prime}{ }_{t} \cdot \mathbf{x}$, where $\mathbf{u}^{\prime \prime}{ }_{t}$ is the projection of $\mathbf{u}^{\prime}$ onto the subspace $E^{\prime \prime}$ spanned by the $\mathrm{x} \in S^{\prime \prime}$.

These considerations allow us to bound $\mathbf{u}^{\prime}$ in term of, only, the margin $\Delta$ and the positive number $\delta^{\prime}$ defined by

$$
-\delta^{\prime}:=\max _{\left|\mathbf{u}^{\prime \prime}\right|=1} \min _{\mathbf{x} \in S^{\prime \prime}}\left\{\mathbf{u}^{\prime \prime} \cdot \mathbf{x}\right\} .
$$

To find such a bound, we decompose a vector $\mathbf{x}^{\prime} \in E^{\prime}$, first, as

$$
\mathbf{x}^{\prime}=\mathbf{x}_{\mathbf{v}}^{\prime}+x^{\prime} \mathbf{v}
$$

with $\mathbf{x}_{\mathbf{v}}$ orthogonal to $\mathbf{v}$; further, we decompose

$$
\mathrm{x}_{\mathbf{v}}^{\prime}=\mathrm{x}^{\prime \prime}+\mathrm{x}_{\mathrm{n}}^{\prime},
$$

with $\mathbf{x}^{\prime \prime} \in E^{\prime \prime}$ orthogonal to $\mathbf{x}_{\mathrm{n}}$. That is,

$$
\mathbf{x}^{\prime}=\mathbf{x}^{\prime \prime}+\mathbf{x}_{\mathrm{n}}^{\prime}+x^{\prime} \mathbf{v}
$$

For a $\mathbf{x}_{t}$ that minimizes $\mathbf{u}_{t}^{\prime} \cdot \mathbf{x}^{\prime}$, we have:

a) either $\mathbf{x}_{t} \in S^{\prime \prime}$. In this case it also minimizes $\mathbf{u}_{t}^{\prime \prime} \cdot \mathbf{x}$, and therefore, $\mathbf{u}_{t}^{\prime \prime} \cdot \mathbf{x}_{t} \leq-\delta^{\prime}\left|\mathbf{u}_{t}^{\prime \prime}\right|$;

b) or $\mathbf{x}_{t} \in S_{\mathrm{c}}^{\prime \prime}$. In this case $x_{t} \geq \Delta$ and therefore, $\mathbf{u}_{t}$. $\mathbf{x}_{t} \leq 0$ implies $\mathbf{u}_{t \mathbf{v}}^{\prime} \cdot \mathbf{x}_{t \mathbf{v}}^{\prime} \leq-\Delta u_{t}^{\prime}$.

Now, if $\mathbf{x}_{t}$ visits $S^{\prime \prime}$, only the component $\mathbf{u}_{t}^{\prime \prime}$ changes, and it actually decreases if $\left|\mathbf{u}_{t}^{\prime \prime}\right|>1 / 2 \delta^{\prime}$. If $\mathbf{x}_{t}$ visits $S_{\mathrm{c}}^{\prime \prime}$, the component $u_{t}^{\prime}$ grows at least by $\Delta$. Therefore, after at most

$$
\beta=\frac{1}{\Delta}\left(\frac{1}{\Delta}-u_{t_{\alpha}}\right)
$$

visits to $S_{\mathrm{c}}^{\prime \prime}$, the component $\left|\mathbf{u}_{t \mathbf{v}}^{\prime}\right|$, and in consequence $\left|\mathbf{u}_{t}^{\prime \prime}\right|$ and $\left|\mathbf{u}_{t \mathrm{n}}^{\prime}\right|$, will stop increasing due to visits to $S_{\mathrm{c}}^{\prime \prime}$. Thus, the following bounds hold

$$
\begin{aligned}
& \left|\mathbf{u}_{t}^{\prime \prime}\right| \leq \max \left\{\left|\mathbf{u}_{t_{\alpha}}^{\prime \prime}\right|+\beta, \frac{1}{2 \delta^{\prime}}+\beta+1\right\} \\
& \left|\mathbf{u}_{t \mathrm{n}}^{\prime}\right| \leq\left|\mathbf{u}_{t_{\alpha} \mathrm{n}}^{\prime}\right|+\beta
\end{aligned}
$$

From the above bounds, we find a bound to $\left|u_{t}^{\prime}\right|$ as follow. After step $t_{\alpha}, x_{t}^{\prime}$ is either zero or bigger than $\Delta$, since only vectors of $S^{\prime}$ are chosen. If $u_{t}^{\prime}=u_{t-1}^{\prime}+x_{t-1}^{\prime} \leq 0$, from step $t-1$ to step $t$, the absolute value of $u^{\prime}$ decreases, hence

$$
\left|u_{t}^{\prime}\right| \leq\left|u_{t_{\alpha}}^{\prime}\right|
$$

if $u_{t}^{\prime}>0$,

$$
\begin{aligned}
0 \geq \mathbf{u}_{t} \cdot \mathbf{x}_{t}=\mathbf{u}_{t}^{\prime} \cdot \mathbf{x}_{t}^{\prime}= \\
\mathbf{u}_{t \mathbf{v}}^{\prime} \cdot \mathbf{x}_{t \mathbf{v}}^{\prime}+u_{t}^{\prime} x_{t}^{\prime} \geq-\left|\mathbf{u}_{t, \mathbf{v}}^{\prime}\right|+u_{t}^{\prime} \Delta
\end{aligned}
$$

Therefore,

$$
\left|u_{t}^{\prime}\right| \leq \max \left\{\left|u_{t_{\alpha}}^{\prime}\right|, \frac{1}{\Delta}\left|\mathbf{u}_{t, \mathbf{v}}^{\prime}\right|\right\} .
$$

It was shown above that $\left|\mathbf{u}_{t}\right|$ is overall bounded by a number that depends only on the data instance $S$. By setting

$$
\begin{gathered}
u^{*}=\sup _{t}\left\{\left|\mathbf{u}_{t}\right|\right\} \\
u=\sup \left\{u^{*}\right\}
\end{gathered}
$$

we finally obtain

$$
\frac{\left|\mathbf{c}_{t}-\mathbf{c}\right|}{R} \leq \frac{u^{*}}{t} \leq \frac{u}{t}
$$

To finish the Section, we show by a simple example that $\mathcal{O}(1 / t)$ is the best converge rate achieved by the BadoiuClarkson algorithm in a worst case scenario. 
Example. Let $S=\{-1,1\}$. In this case

$$
u_{t}=0, \pm 1,0, \mp 1,0, \pm 1, \ldots
$$

and,

$$
c_{t}=0, \pm 1, \mp \frac{1}{3}, 0, \pm \frac{1}{5}, \ldots
$$

Hence, $c_{t}$ converges against the origin at a rate exactly $1 / t$, for this instance of the problem. Note also that there is a non-countable number of possibilities for the sequence $\left(u_{t}\right)$ but all of them are bounded by one.

\subsection{On a bound on $u^{*}$}

Computer experiments and intuition, but not yet a proof, suggest that $u^{*}=\max _{t}\left|\mathbf{u}_{t}\right|$ is always bounded by

$$
u^{*} \leq \sqrt{2 d} .
$$

In most of the cases which we have encountered, this is a rather conservative bound. The next section will take a closer look at it. If $n \leq d$, everything happens in a subspace of at most $n$ dimensions. In this case $u^{*} \leq \sqrt{2 n}$. Combined this yields as the proposed bound for $u$

$$
u \leq \min \{\sqrt{2 d}, \sqrt{2 n}\}
$$

\section{Empirical Results}

For all test sets, two random points were pre-specified to lie on opposite sites, respectively, to guarantee for the points to span the intended hypersphere and therefore to ensure for a known ground truth for the precision measurement. In all three scenarios the convergence follows a line of slope -1 , which demonstrates the $\mathcal{O}(1 / t)$ convergence we have proven.

In a first trial, we generated 5.000 points in 100 dimensions randomly distributed on a ball of unit radius. Even though this is a straightforward model, we have a more or less untypical situation in which all points are potential attractors for the center. In a real-life scenario, it is very likely to have only few points that are actually located on the SEB. Anyhow, in Figure 2 (top), it can be nicely seen, how the slope of these points is clung to the $\mathcal{O}(1 / t)$ slope.

Then, we distributed 100 points in 100 dimensions randomly on the vertices of a unit hypercube. This distribution appears to be much more challenging for the algorithm due to the equally distributed points on the edges of the cube. But still, after a finite number of steps, the slope clings to the line of slope -1 , as it can be seen in Figure 2 (middle).

The third scenario illustrates the worst case, on which the estimation is based for the step size of the learning rule in Formula (1). For 100 dimensions, we took 100 linear independent points that are all located on the same hemisphere
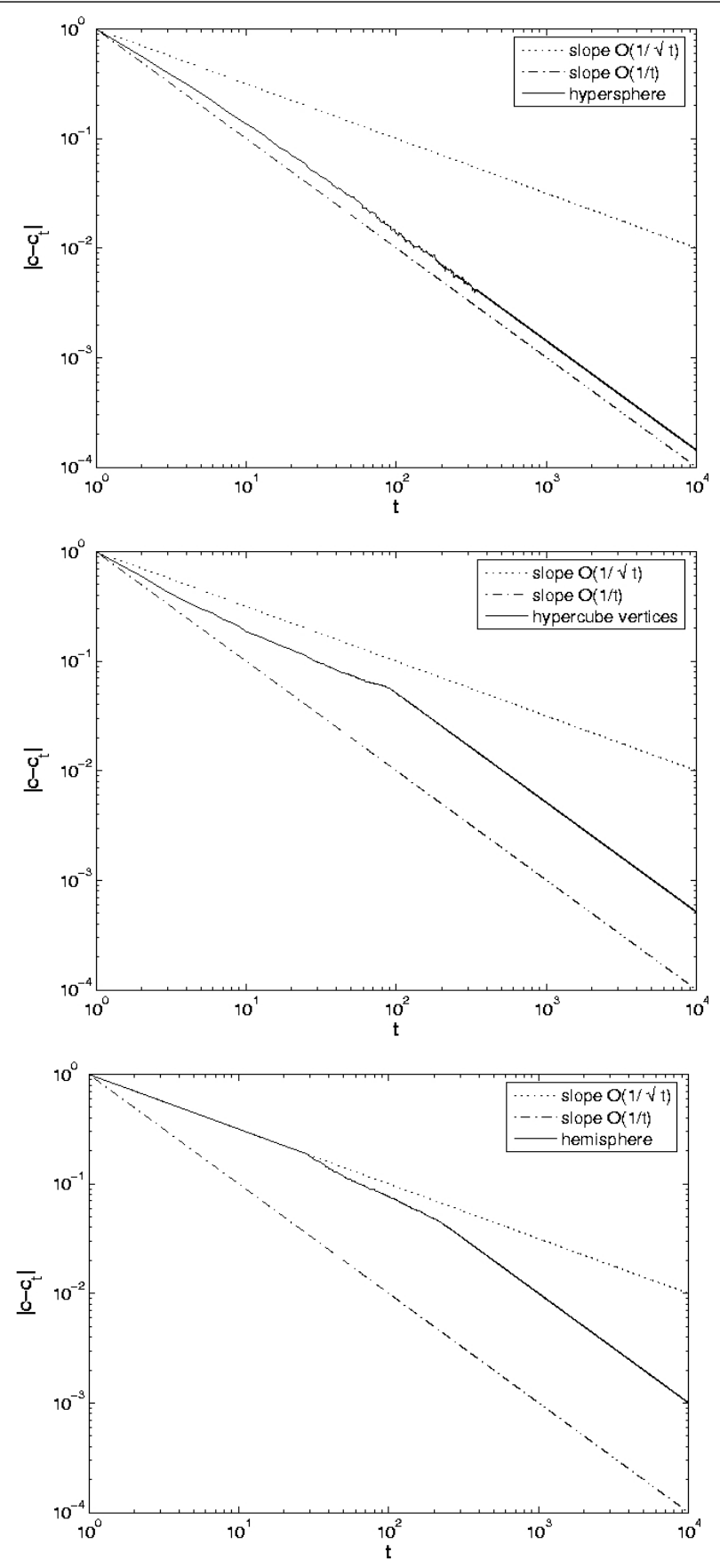

Figure 2. Deviation from the exact center $x$ number of iterations (double-log plot). Top: $n=5000, d=100$ randomly distributed on an unity ball. Middle: $n=100, d=100$ on a hypercube. Bottom: $n=101, d=100$, all but one orthogonal and on a single hemisphere. Deviation decreases to zero along a line of slope -1 , as proven. The line of slope $-1 / 2$ gives the old upper bound. 
of a unit hypersphere and only one point in the other half. But again, in Figure 2 (bottom), it can be seen that after a finite number of steps the slope changes to the proposed $\mathcal{O}(1 / t)$ convergence.

\subsection{The proposed bound for $u^{*}$}

To test a bound on the constant $u^{*}$ at least empirically, we calculated many SEB with Badoiu and Clarkson's algorithm in different dimensions and with different number of points:

a) randomly distributed on a ball of unit radius;

b) randomly distributed on the vertices of a unit hypercube;

c) and orthogonal points located on a quadrant with only one point on other adjacent quadrant (analog to the two scenarios above).

For each run we determined the $u^{*}$ which occurred and compared it with the assumed bound. In Figure 3, these $u^{*}$ values (normalized by $\sqrt{2 d}$ ) are shown for the first two scenarios. The dimension varied from 2 to 32 , and in each dimension the number of points $n$ varied from 2 to $4 d$, resulting in about $170.000 u^{*}$ values. The line in Figure 3 indicates the bound which must not be exceeded. In both scenarios the $u^{*}$ values are largest around $n=d$. For $n<d$ the effective dimension decreases with decreasing $n$ and, hence, the task becomes easier. For $n>d$ the task becomes easier with increasing $n$ since the probability increases that $\mathbf{u}_{t} \cdot \mathbf{x}_{t}$ is largely negative with an iteration step, which keeps $u^{*}$ small, see Equation (8). Indeed, a few $u^{*}$-values come close, but none exceeds the proposed bound.

As the number of points in the hemisphere scenario $c$ is bound to $d+1$ points, the corresponding scatter plot is not very illustrative, but the proposed bounds have not been exceeded in any case.

Since the value $u$ depends only on the data instance, the algorithm is expected to be $\mathcal{O}(1 / t)$ at least in average. While it has not been shown yet, the expected value for $u$ can be inferred by sampling. Figure 4 shows how the sample means $\mu$ depends on $\min \{\sqrt{2 n}, \sqrt{2 n}\}$ for scenario $a$ ) and $b$ ). The curves $\mu \pm 1.96 \sigma$, (where $\sigma$ is the standard deviation of the samples) and the proposed bound are also shown. Note that the curve $\mu+1.96 \sigma$ is always below the proposed bound.

\section{Summary}

We have shown that for a given set $S$, Badoiu and Clarkson's algorithm converges at least as $\mathcal{O}(u / t)$ to the correct SEB. Intuition and computer experiments, but not yet a proof, suggest that always $u \leq \min \{\sqrt{2 d}, \sqrt{2 n}\}$ is valid.
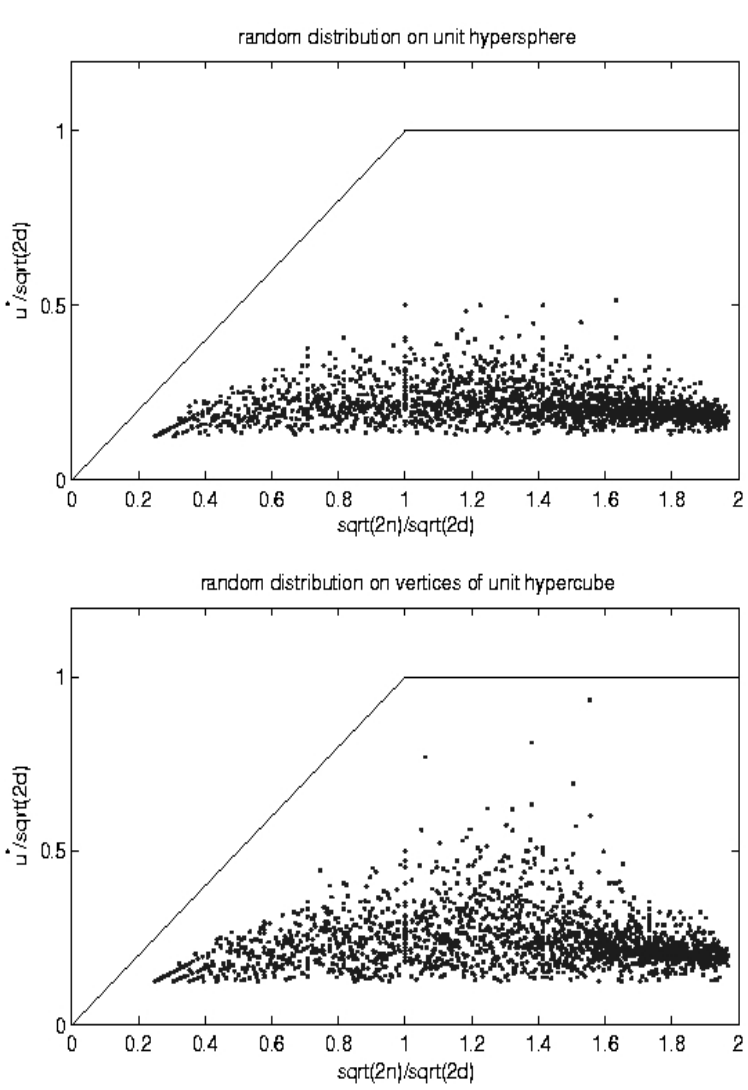

Figure 3. The $u^{*}$-values (dots) we obtained in about 170.000 calculations of SEB in $d=$ $2,3, \ldots, 32$ dimensions and with $n=2, \ldots, 4 d$ points in two different scenarios. Top: randomly distributed on a hypersphere. Bottom: randomly distributed on the vertices of a hypercube. The proposed bound is indicated by the line. This bound holds in all tested configurations. On average, $u^{*}$ is smaller for the hypersphere scenario.

In case this bound holds, Badoiu and Clarkson's simple algorithm finds the smallest enclosing ball around points with $\epsilon$ precision in at most

$$
\mathcal{O}\left(\min \left\{\frac{n d^{3 / 2}}{\epsilon}, \frac{n^{3 / 2} d}{\epsilon}, \frac{n d}{\epsilon^{2}}\right\}\right)
$$

time. Hence, with the other quantities fixed, asymptotically the computational time increases like $\mathcal{O}(n), \mathcal{O}(d)$, and $\mathcal{O}(1 / \epsilon)$, respectively. The extension of our convergence analysis of Badoiu and Clarkson's algorithm to smallest enclosing balls around balls (SEBB) is straightforward. 

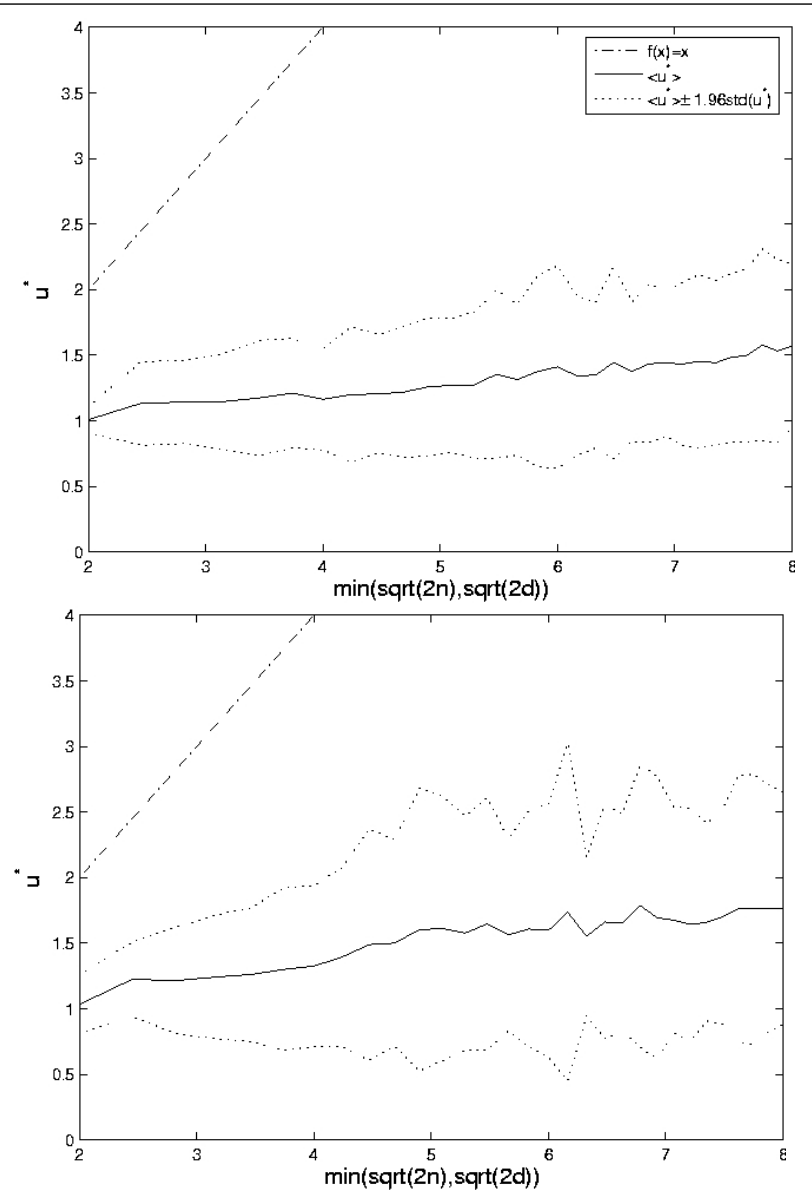

Figure 4. Dependence of the sample means $\mu$ over $\min \{\sqrt{2 n}, \sqrt{2 n}\}$ for two different scenarios. Top: randomly distributed on a hypersphere. Bottom: randomly distributed on the vertices of a hypercube. The curves $\mu \pm 1.96 \sigma$, (where $\sigma$ is the standard deviation of the samples) and the proposed bound are also shown. Note that the curve $\mu+1.96 \sigma$ is always below the proposed bound.

\section{Acknowledgements}

$\mathrm{CM}$ is partially supported by $\mathrm{CNPq} / \mathrm{FAPEAM}$.

\section{References}

[1] M. Badoiu and K. L. Clarkson. Smaller core-sets for balls. Proc. 14th ACM-SIAM Symposium on Discrete Algorithms (SoDA), pages 801-802, 2003.

[2] M. Badoiu, S. Har-Peled, and P. Indyk. Approximate clustering via core-sets. Proc. 34th Symp. Theory of Computing, pages 250-257, 2002.
[3] Y. Bulatov, S. Jambawalikar, P. Kumar, and S. Sethia. Hand recognition using geometric classifiers. DIMACS Workshop on Computational Geometry, 2002.

[4] C. J. C. Burges. A tutorial on support vector machines for pattern recognition. Data Mining and Knowledge Discovery, 2(2):121-167, 1998.

[5] O. Chapelle, V. Vapnik, O. Bousquet, and S. Mukherjee. Choosing multiple parameters for support vector machines. Machine Learning, 46:131-159, 2002.

[6] K. Fischer, B. Gärtner, and M. Kutz. Fast smallest-enclosingball computation in high dimensions. Proc. 11th European Symposium on Algorithms (ESA), pages 630-641, 2003.

[7] A. Goel, P. Indyk, and K. R. Varadarajan. Reductions among high dimensional proximity problems. In Symposium on Discrete Algorithms, pages 769-778, 2001.

[8] J. T. Kim, J. Gewehr, and T. Martinetz. Binding matrix: a novel approach for binding site recognition. Journal of Bioinformatics and Computational Biology, 2:289-307, 2004.

[9] W. Krauth and M. Mezard. Learning algorithms with optimal stability in neural networks. J.Phys.A, 20:745-752, 1987.

[10] P. Kumar, J. S. B. Mitchell, and A. Yildırım. Computing core-sets and approximate smallest enclosing hyperspheres in high dimensions. Algorithm Engineering and Experimentation (ALENEX), Lecture Notes Comput. Sci., pages 45-55, 2003.

[11] A. Madany Mamlouk, J. T. Kim, E. Barth, M. Brauckmann, and T. Martinetz. One-class classification with subgaussians. DAGM 2003, Lecture Notes Comput. Sci., 2781:346-353, 2003.

[12] T. Martinetz. Minover revisited for incremental supportvector-classification. Lecture Notes in Computer Science, 3175:187-194, 2004.

[13] N. Megiddo. Linear-time algorithms for linear programming in $\mathrm{r}$ and related problems. SIAM J. Comput., pages 759-776, 1983.

[14] M. Minsky and S. Papert. Perceptrons. MIT Press, 1969.

[15] E. Welzl. Smallest enclosing disks (balls and ellipsoids). New Results and New Trends in Computer Science, LNCS, 555:359-370, 1991. 